\title{
Patch types in Posidonia oceanica meadows around Corsica How can we use them in seascape ecology?
}
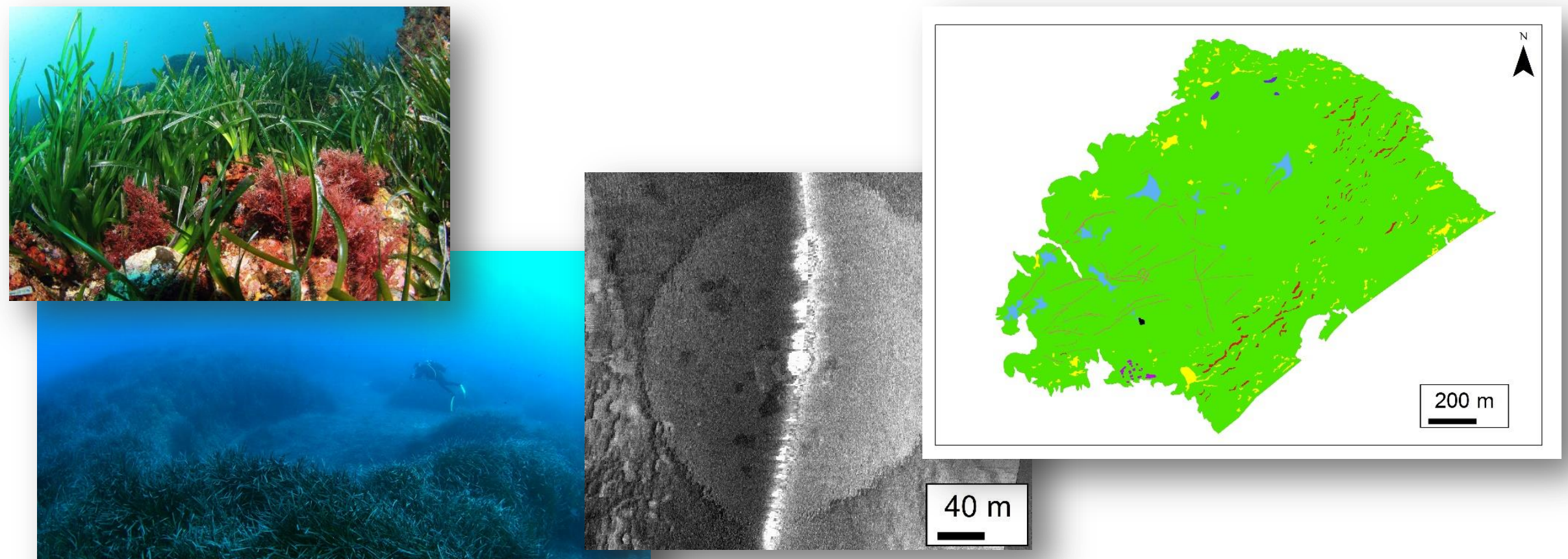

Sardinia, Italy - May 2015

Arnaud Abadie, Marina Bonacorsi, Sylvie Gobert, Pierre Lejeune, Gérard Pergent, Christine Pergent-Martini 


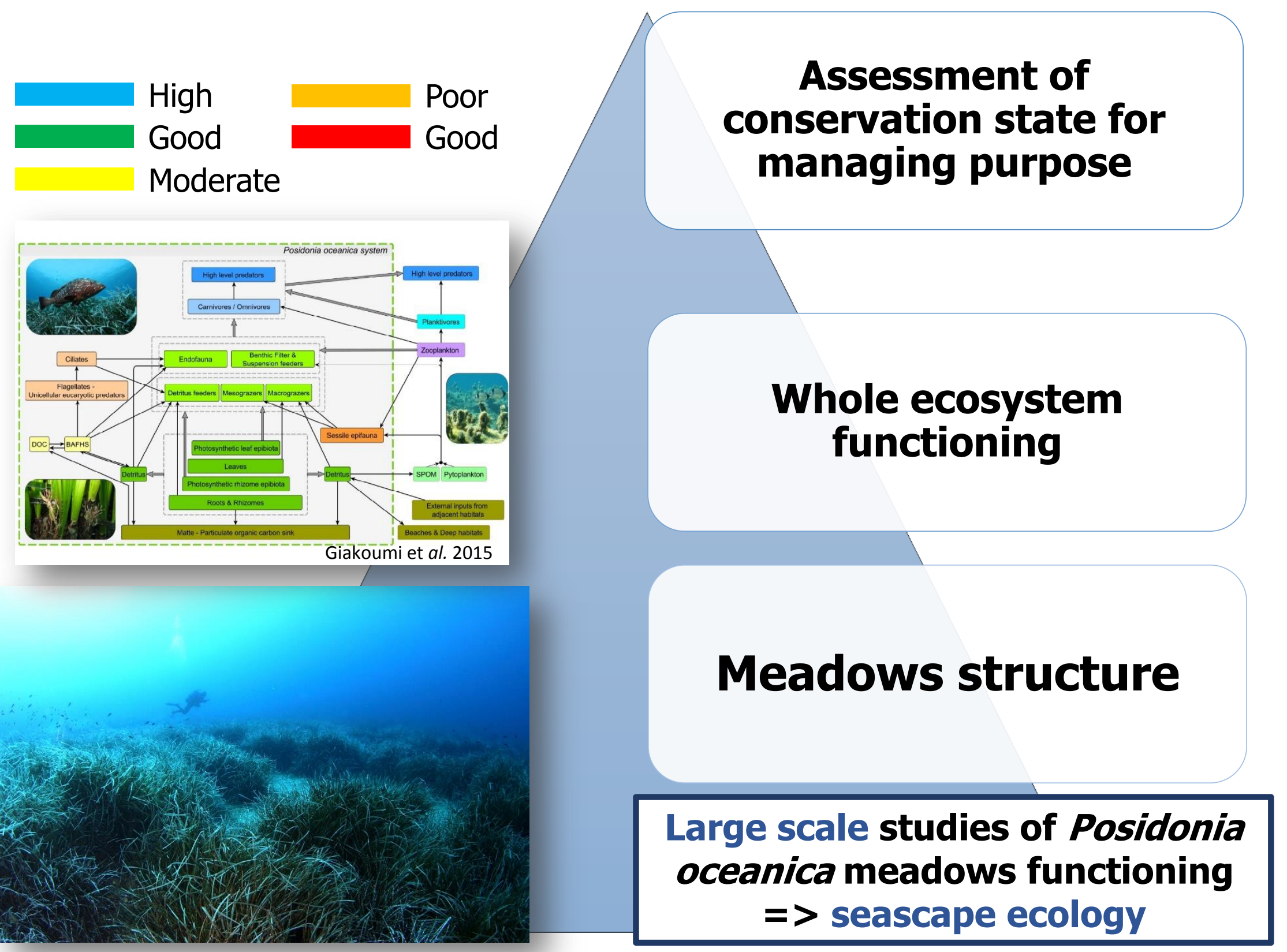




\section{Five sites around Corsica}

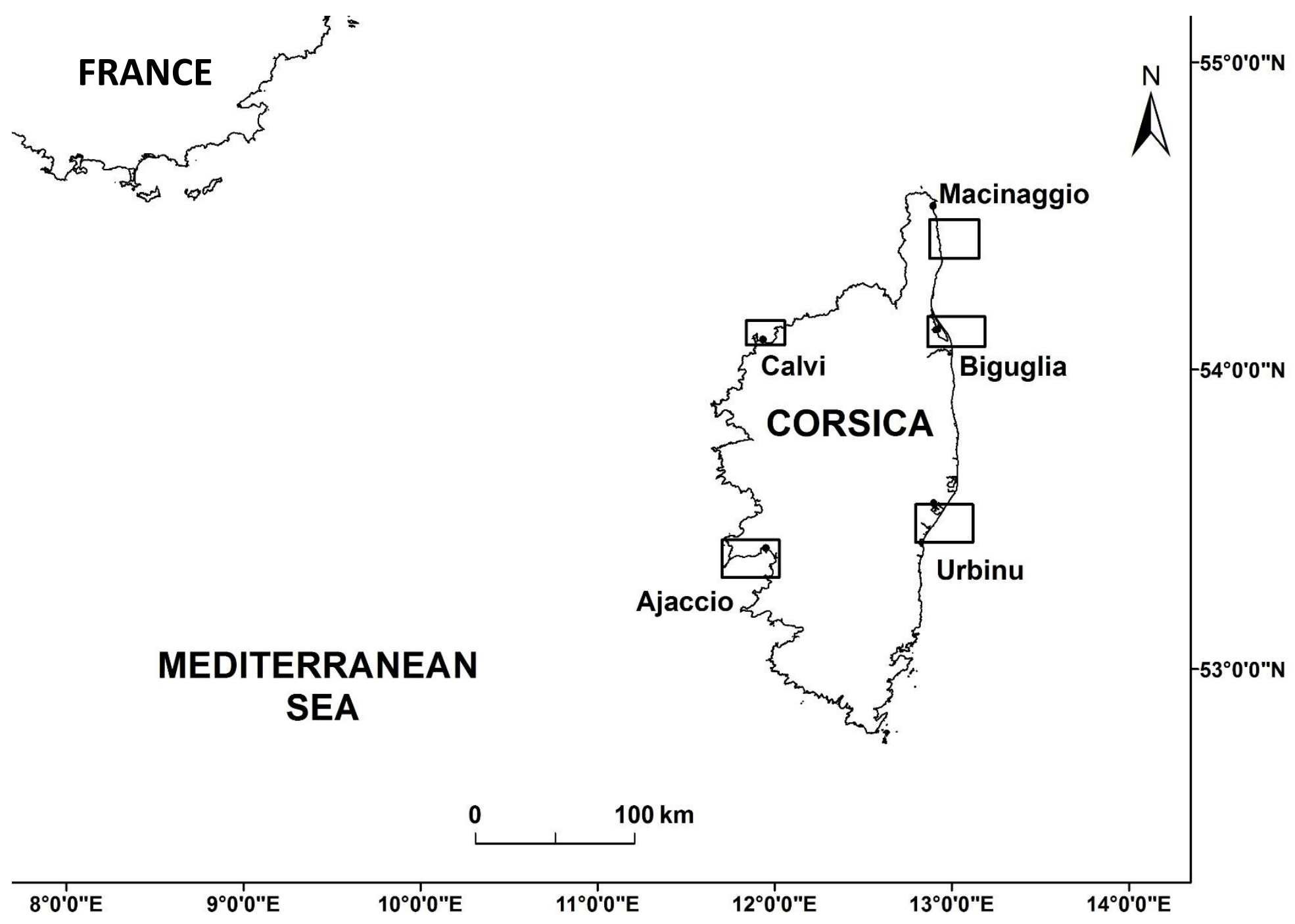




\section{Five sites around Corsica}
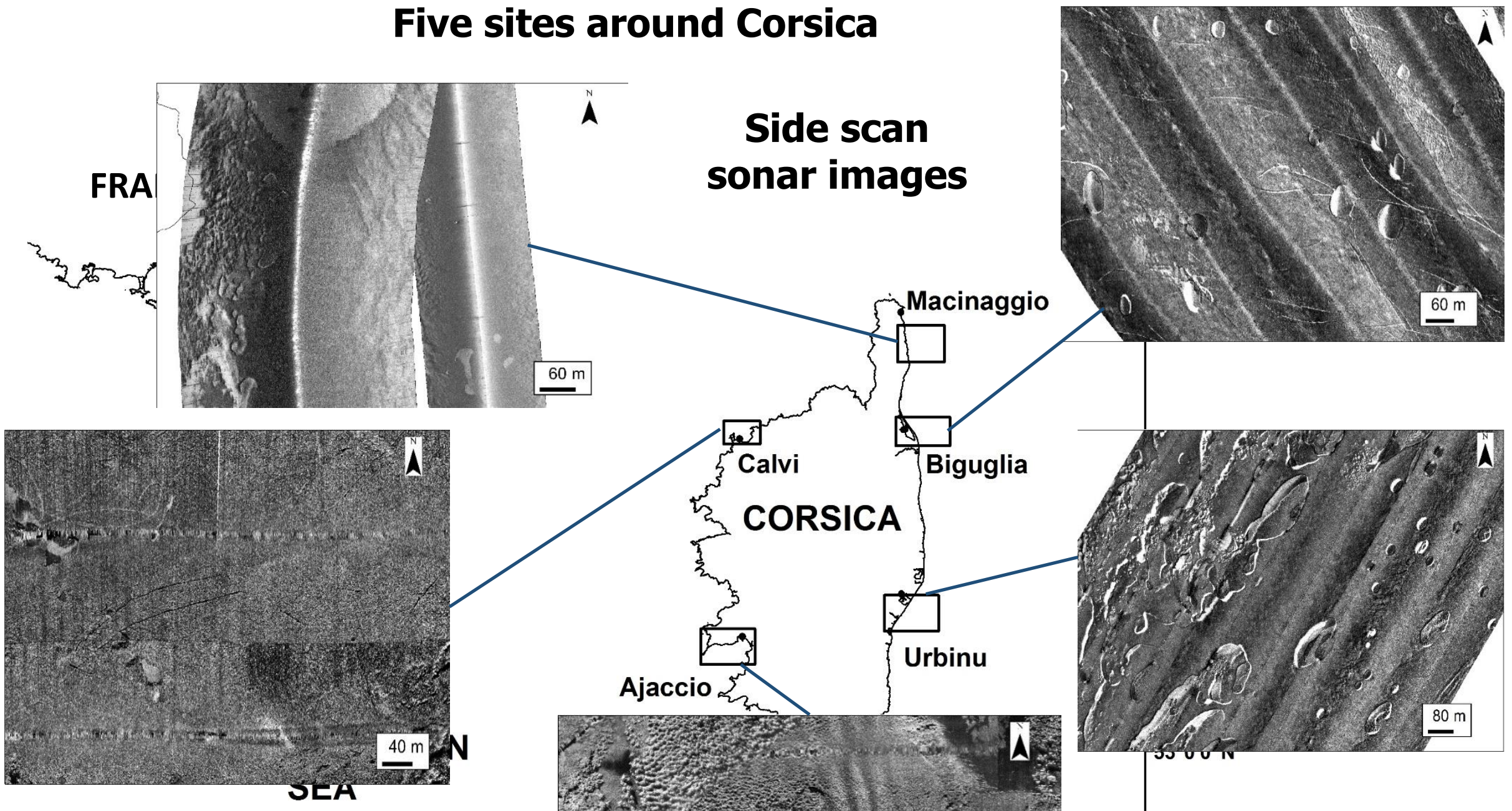
Meadow matrix

Continuous meadow

No patches

No natural or anthropogenic impact 


\section{Heterogeneous meadow}

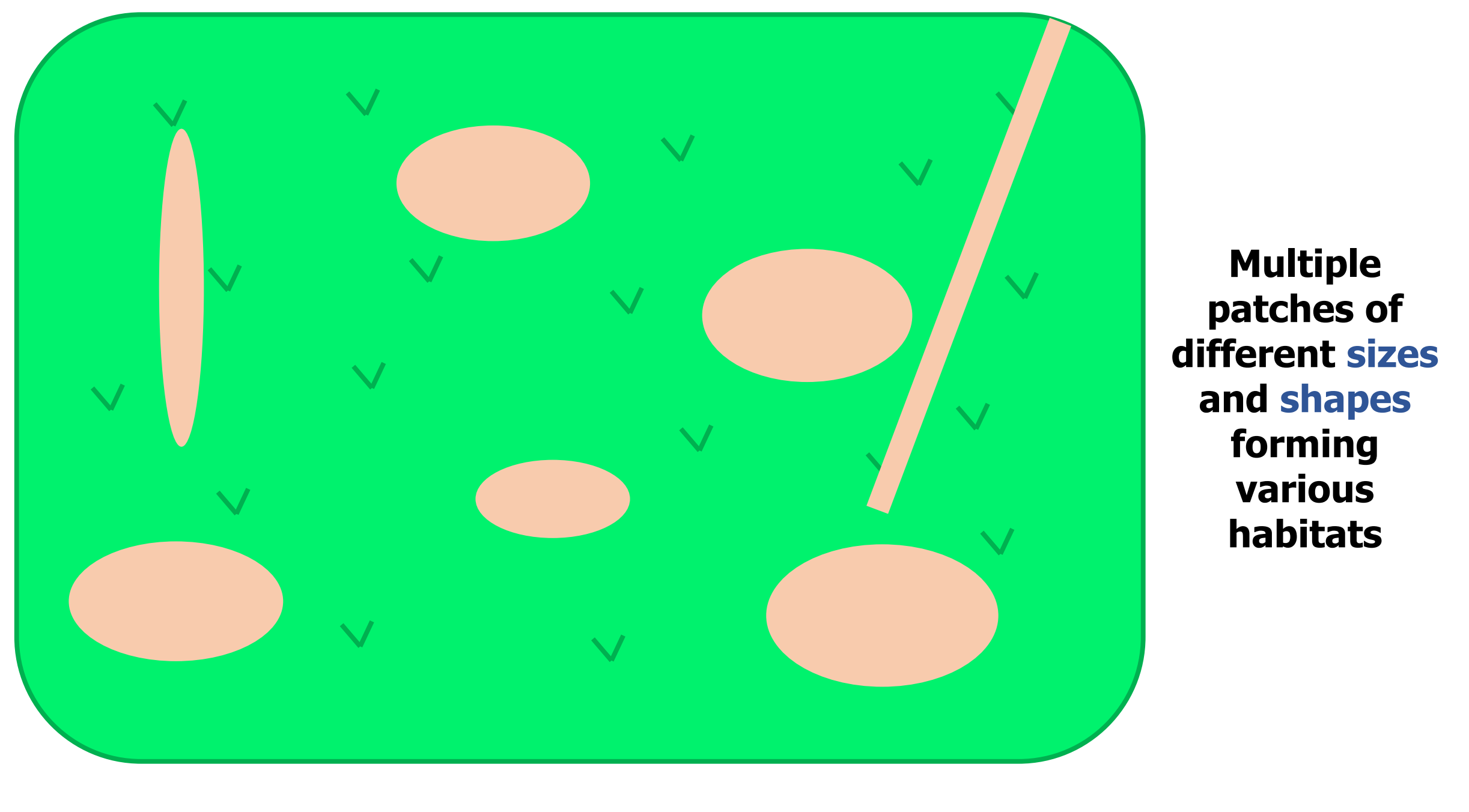




\section{Heterogeneous meadow}

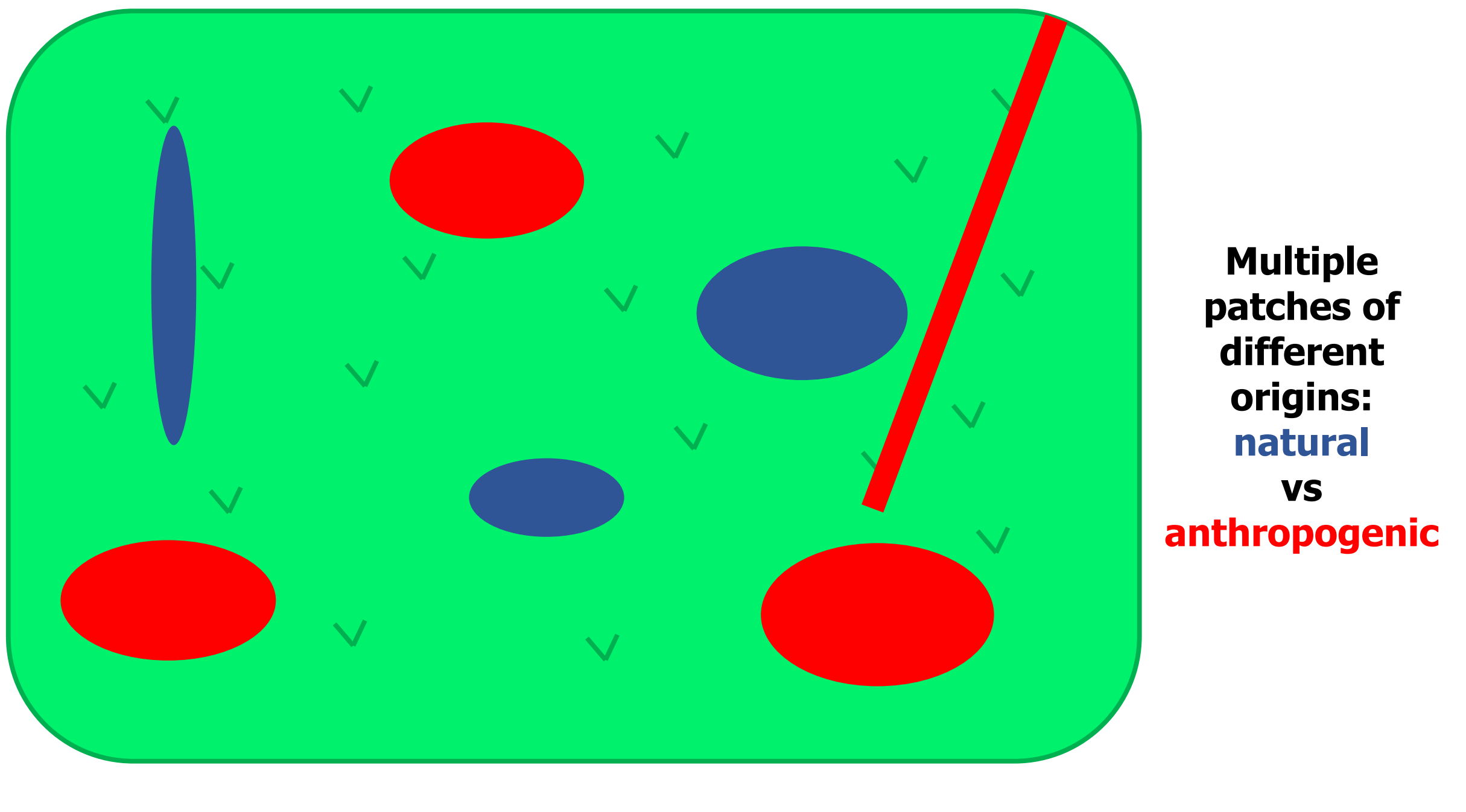




\section{Heterogeneous meadow}

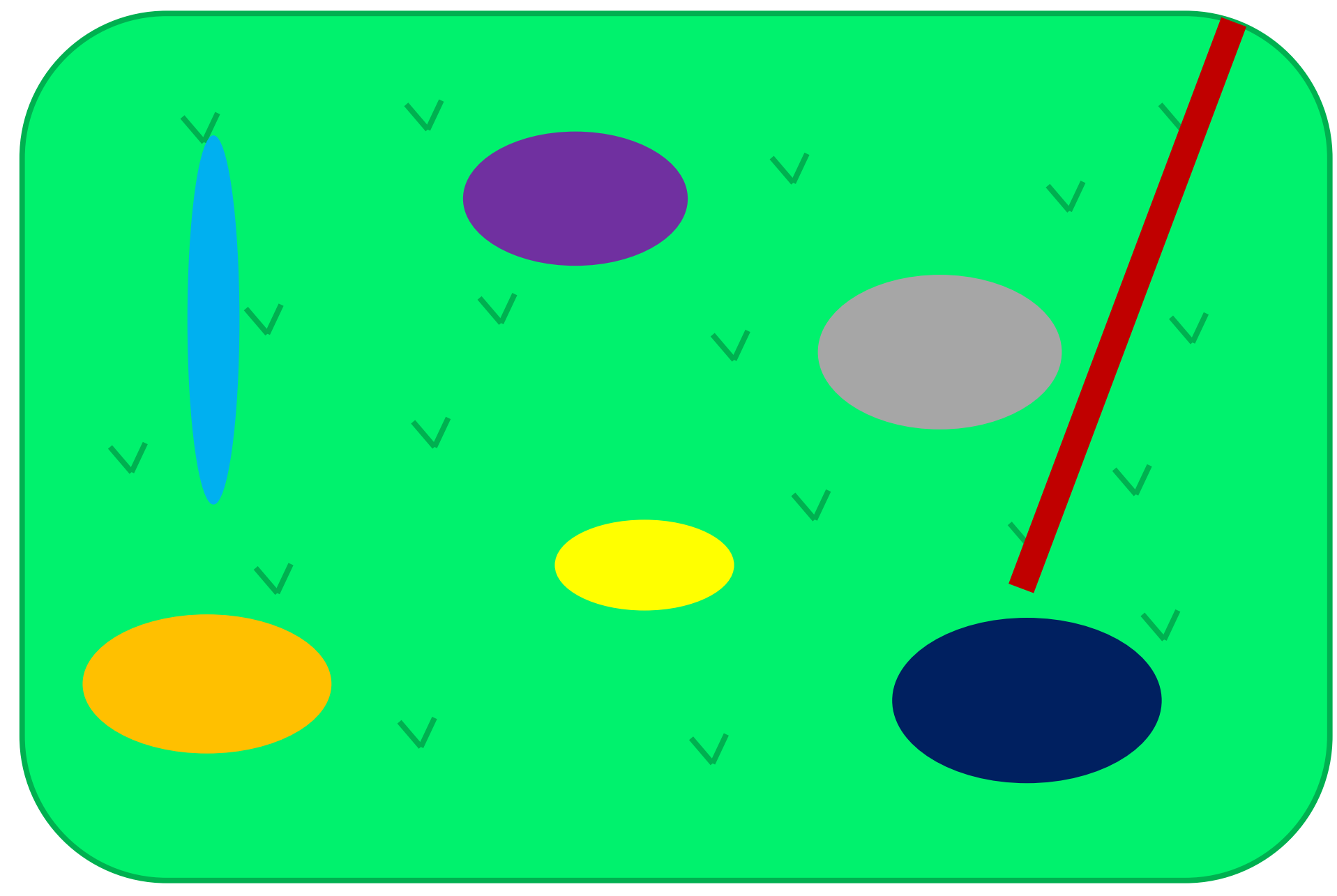

\section{Multiple} patches of various types 


\section{1 types (or classes) of patch identified}

\section{5 natural}

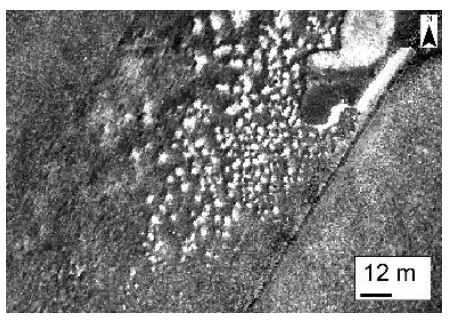

Small sand patch

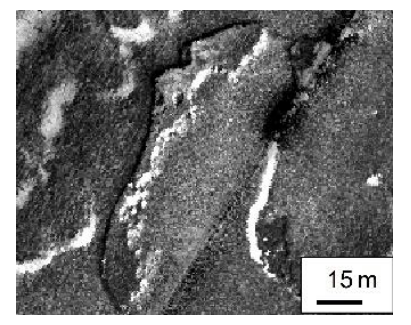

Sand corridor

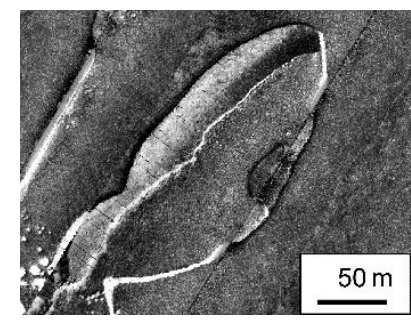

Large sand patch

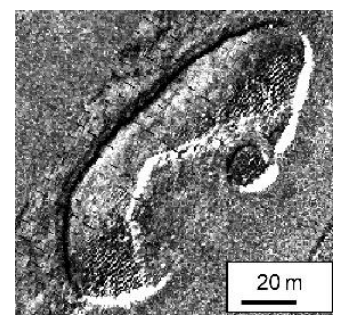

Colonized patch

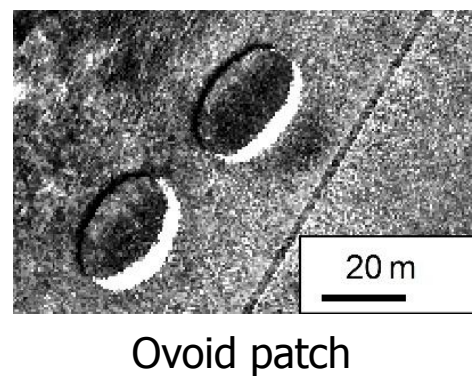

Ovoid patch

\section{5 anthropogenic}

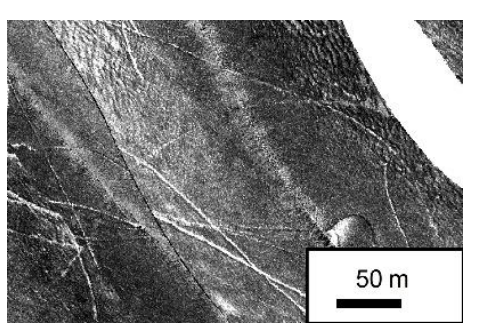

Trawling tracks

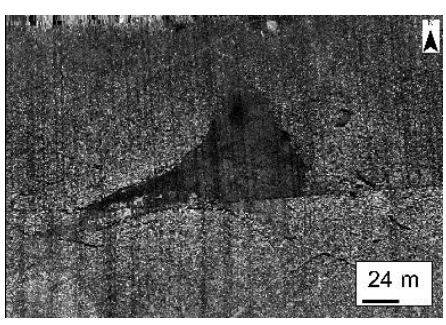

Anchoring patch

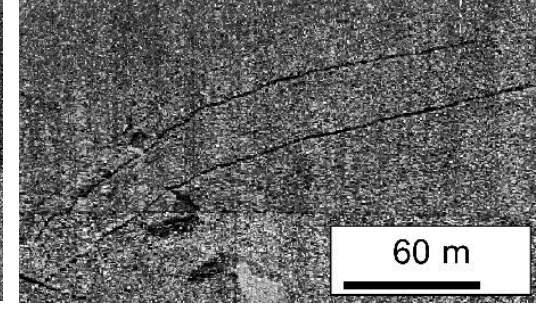

Anchoring track

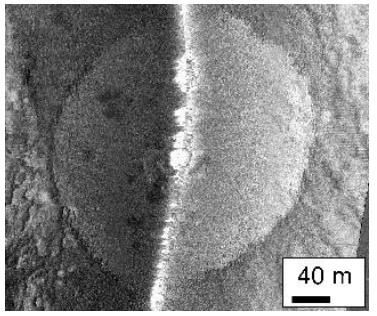

Bomb impact

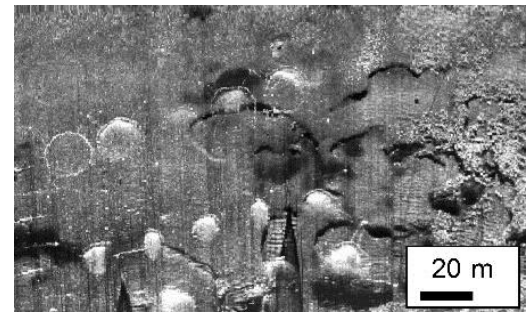

Fish farm patch

\section{1 can be of both origins}

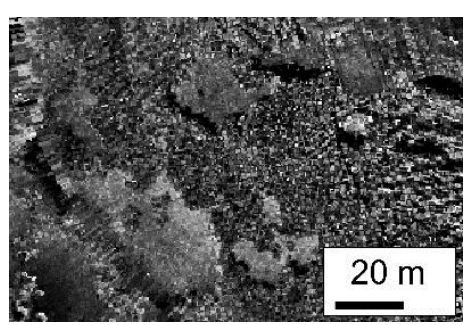

Bare mat 


\section{Biguglia}
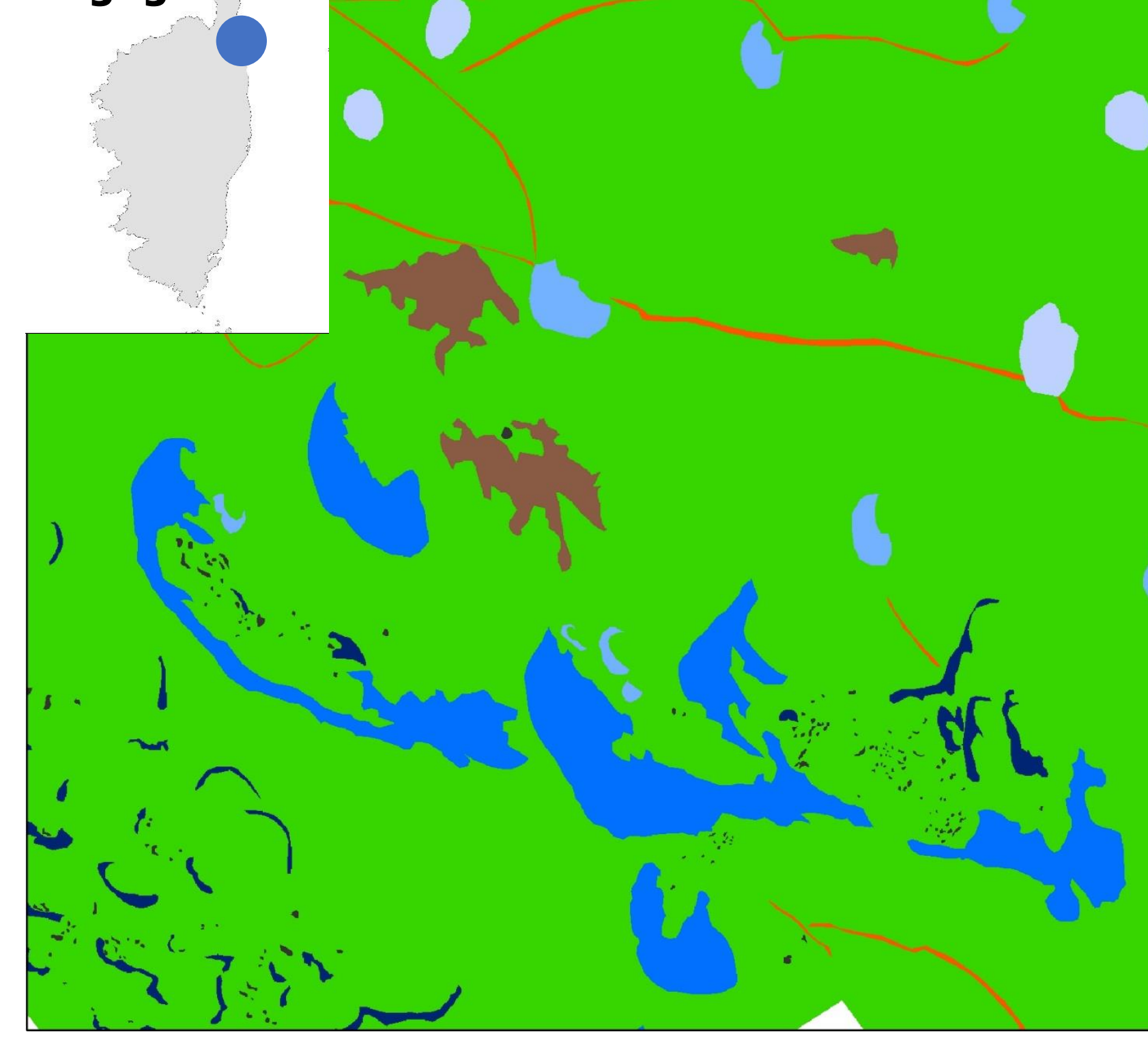


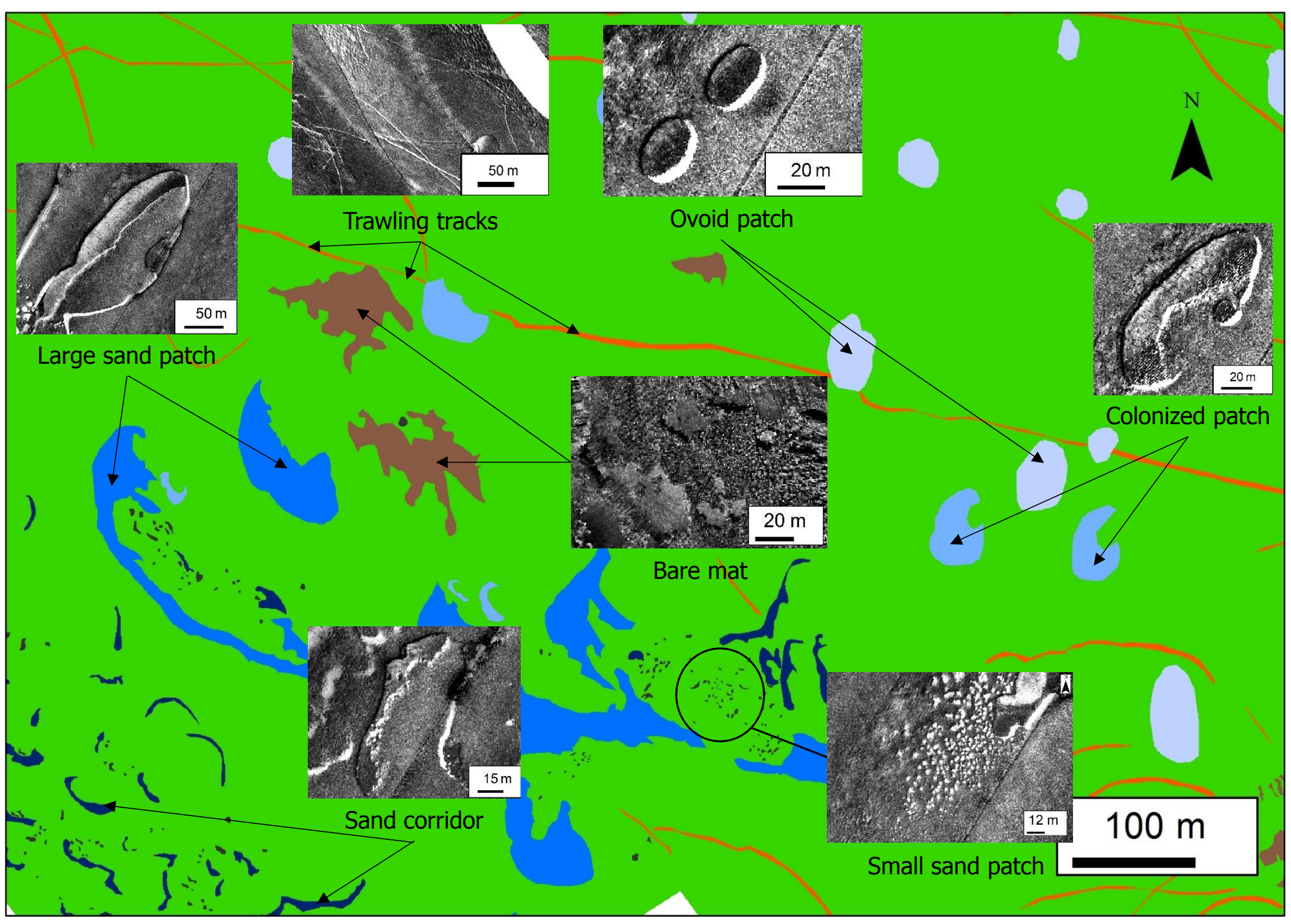




\section{Seascape analysis}

\section{A simple area index}

\section{natural vs anthropogenic}

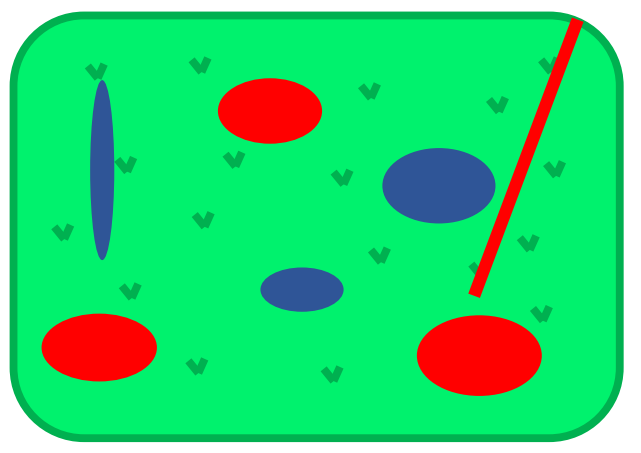

$$
\boldsymbol{P a S I}=\frac{S_{N P}}{S_{N P}+S_{A P}}
$$

$\mathrm{S}_{\mathrm{NP}}$ : surface of natural patches $\mathbf{S}_{\mathrm{AP}}$ : surface of anthropogenic patches

\begin{tabular}{|c|c|c|c|}
\hline PaSI Value & Description & Color & Site \\
\hline $0.801-1$ & High natural influence on the meadow patchiness & Blue & Urbinu \\
\hline $0.601-0.800$ & Major natural influence on the meadow patchiness & Green & \\
\hline $0.401-0.600$ & $\begin{array}{l}\text { Moderate anthropogenic influence on the meadow } \\
\text { patchiness }\end{array}$ & Yellow & $\begin{array}{l}\text { Ajaccio, South } \\
\text { Macinaggio, } \\
\text { Biguglia }\end{array}$ \\
\hline $0.201-0.400$ & Major anthropogenic influence on the meadow patchiness & Orange & Calvi \\
\hline $0-0.200$ & High anthropogenic influence on the meadow patchiness & Red & \\
\hline
\end{tabular}




\section{Seascape analysis} patch types

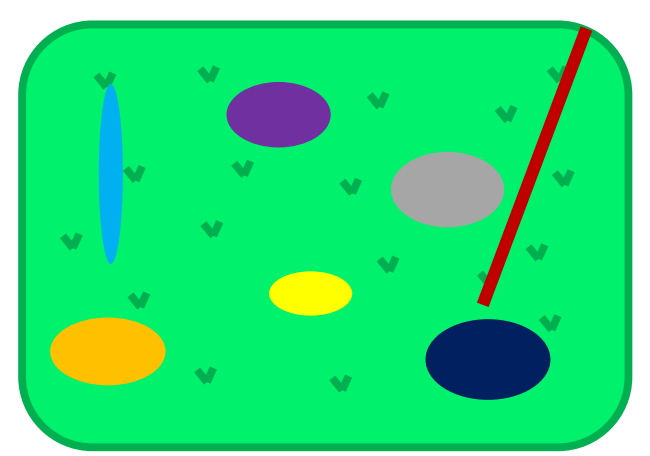

Several patch classes discriminated by their characteristics
Seven landscape metrics + a water movement index (REI) used at patch class level

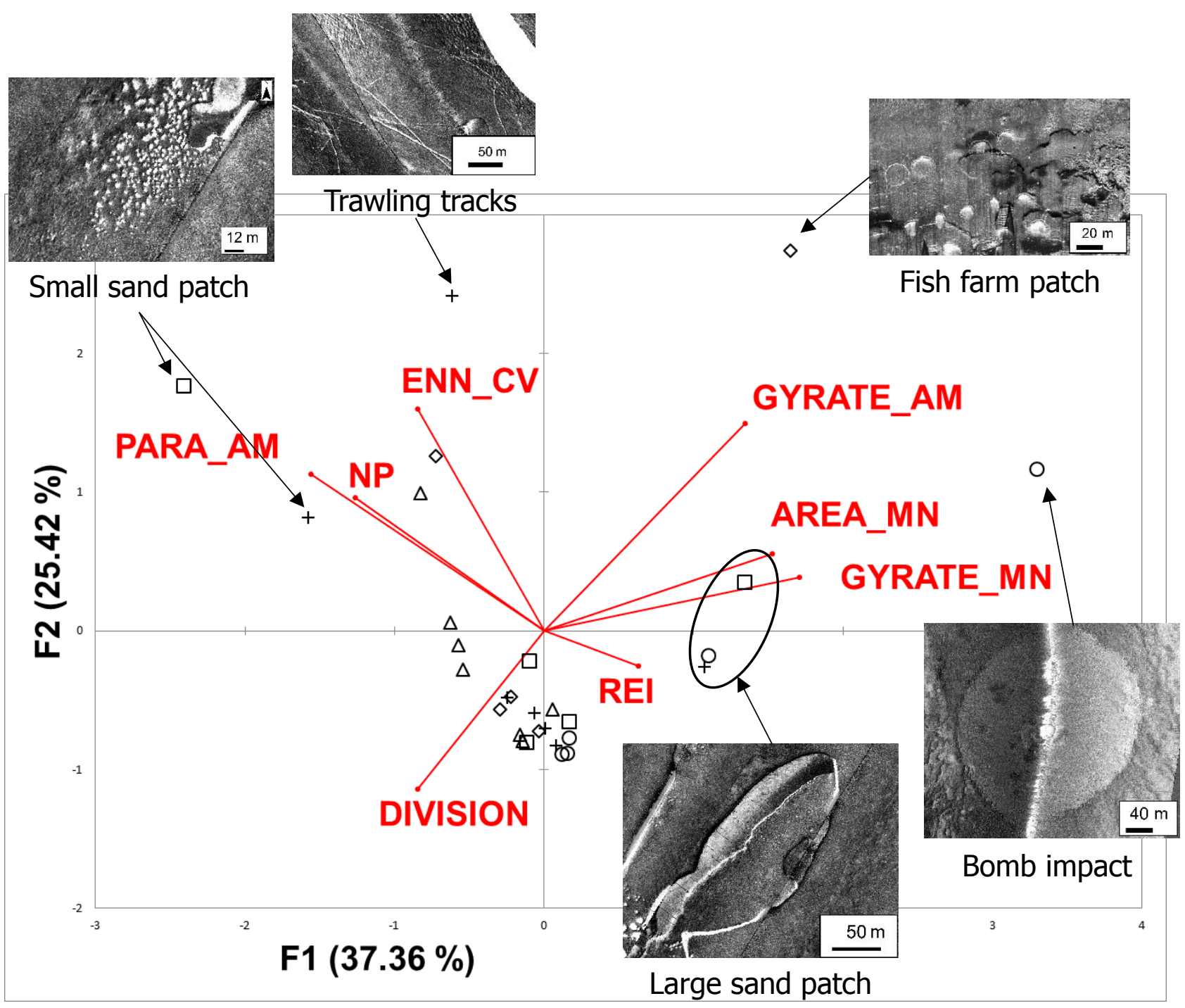




\section{Take-home message}

- 11 patch classes recognizable with sonar images

- P. oceanica seascapes structured by natural and anthropogenic patches

- A great potential use of patch classes characteristics to compute new conservation indices

\section{Going further}

- Combine structure and function at large scale

- 3D maps and analyses of seascapes

- Use of the most recent landscape ecology tools (e.g. landscape graphs)

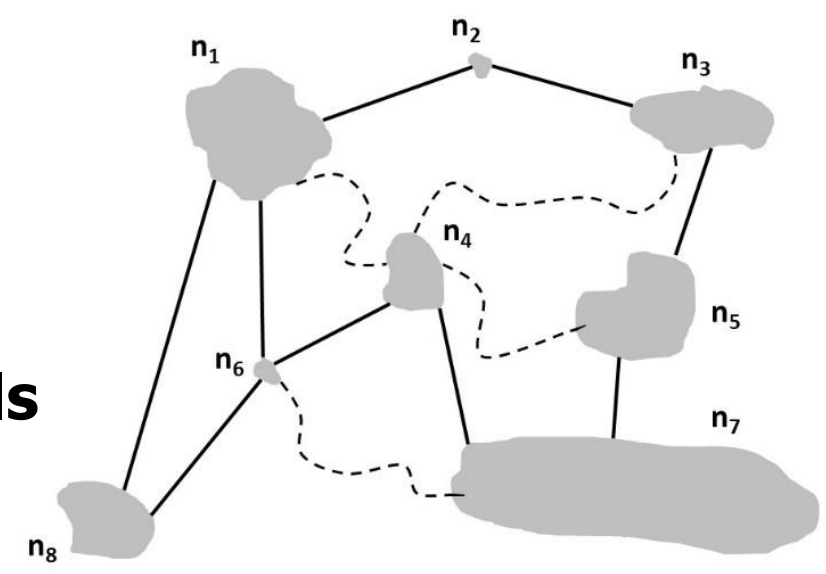


